\title{
Los debates online como metodología docente innovadora en la Universidad
}

\author{
Jesús de la Torre Laso ${ }^{a}$, Rodrigo Morchón García ${ }^{b}$ y José Manuel Fernández Ábalos ${ }^{c}$ \\ ${ }^{a}$ Departamento de Psicología Social. Facultad de Psicología. Universidad de Salamanca (jesutl@usal.es), ${ }^{b}$ Área de

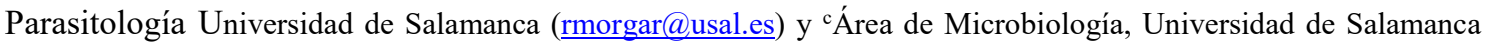 \\ (fernandez.abalos.jm@usal.es).
}

\section{\$EWBDFW}

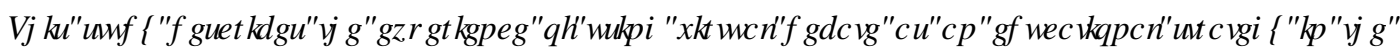

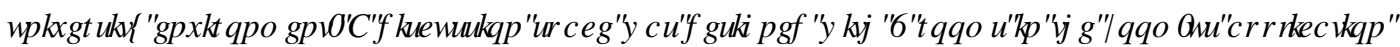

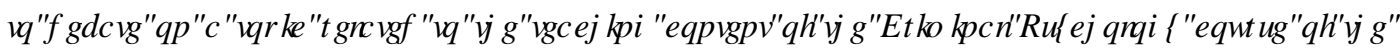

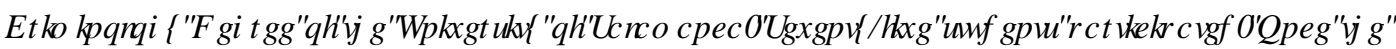

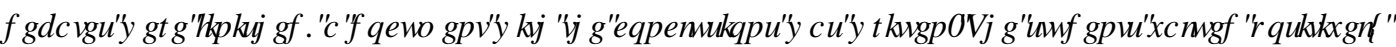

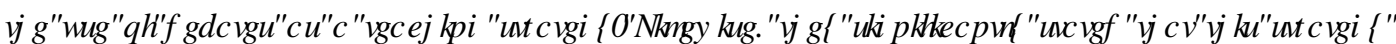

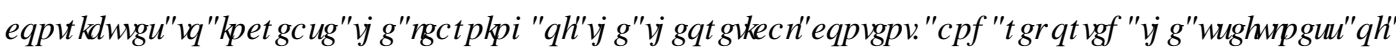
FRQUQXIQJ ZZ LKIUKLVHEXFDURQDOS WFWFHI

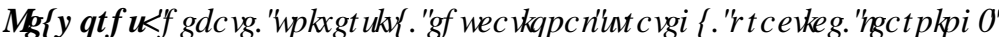

[

\begin{abstract}
5 HXP HQ

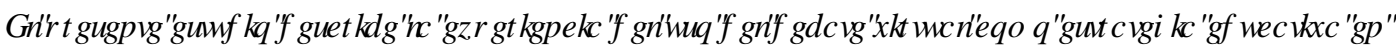

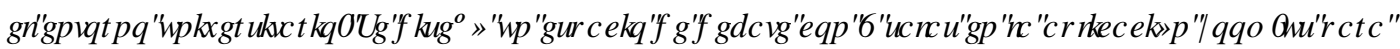

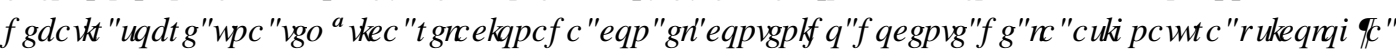

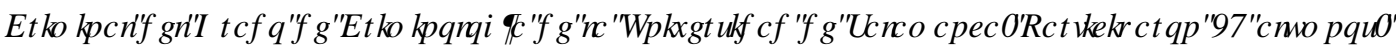

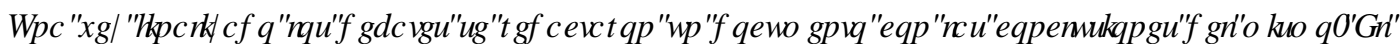

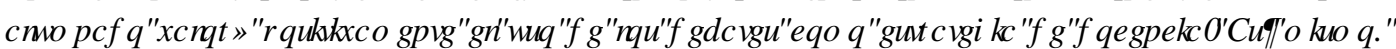

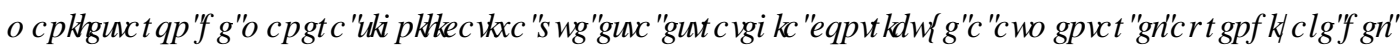

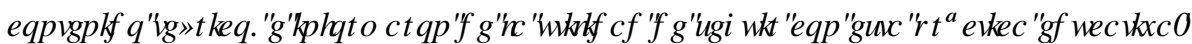

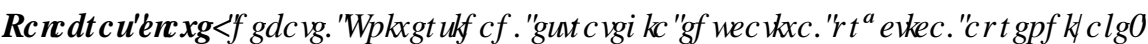

\section{Introducción}

El Espacio Europeo de Educación Superior ha supuesto un rediseño de metodología de enseñanza y las estrategias educativas.

Por un lado, se ha pretendido cambiar el enfoque del proceso enseñanza-aprendizaje mediante el cambio de rol en el alumnado (Marqués Graells, 2001) al situarlo en el centro del proceso de enseñanza-aprendizaje.

Por otro lado, el cambio metodológico ha venido acompañado por la utilización de nuevos recursos tecnológicos. El uso de las Tecnologías de la Información y de la Comunicación (TIC) se han ido incorporando en nuestras universidades, a menudo asociadas a prácticas docentes directivas y poco 
participativas. Aun así, no parece que las prácticas docentes en la enseñanza hayan cambiado de forma evidente (Bernal, 2009). Existe un desfase entre la potencialidad de las TIC's incorporadas en las aulas y la escasa renovación de los procesos pedagógicos (Esteve, 2016). Las TIC's se han ido incorporando en nuestras universidades, a menudo asociadas a prácticas docentes directivas y poco participativas. En otras ocasiones, la incorporación de estrategia docentes innovadoras como el e-portfolio (Wielenga \& Melisse, 2000), el uso de aplicaciones móviles (Morchón García et al., 2016), video tutoriales (Bengochea \& Medina, 2013), las Flipped Classroom (Sola Martínez et al., 2019) y las estrategias con Realidad Aumentada (Maceiras et al., 2010).

La herramienta docente del debate como estrategia docente en las universidades ha sido utilizada como actividad complementaria a la formación académica (Orihuela Gallardo \& Sierra-Casanova, 2020). Así mismo, el uso de esta estrategia docente se ha consolidado como herramienta de aprendizaje cooperativo en la educación superior constituye una estrategia útil y adecuada para la mejora de los aprendizajes (Esteban García \& Ortega Gutiérrez, 2017).

Aceptar la metodología del debate como estrategia docente implica considerar que la enseñanza superior debe ir más allá de la transmisión de conocimientos hacia un modelo de formación integral del alumnado, mediante el desempeño de competencias transversales.

La mayoría de las experiencias sobre los debates se basan en el desarrollo de una discusión entre grupos sobre un mismo tema, con el objeto de trabajar y afianzar diferentes competencias transversales (Tabla 1)

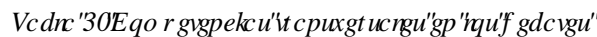

\begin{tabular}{|l|}
\hline \multicolumn{1}{|c|}{ Competencias instrumentales } \\
\hline Capaciadad de análisis y síntesis \\
\hline Capaciadad de organizar y planifica \\
\hline Comunicación oral y escrita \\
\hline Habilidades de gestión de la infomación \\
\hline Resolución de problemas \\
\hline Toma de decisiones \\
\hline \\
\hline Capaciadad de crítica y autocrítica \\
\hline Trabajo en equipo \\
\hline Habilidades inerpersonales \\
\hline Capaciadad de comunicarse con expertos de otras áreas \\
\hline Apreciación de la diversidad y multuculturalidad \\
\hline Compromiso ético \\
\hline \multicolumn{1}{|c|}{ Competencias sitémicas } \\
\hline Capaciadad para aplicar los conocimientos en la práctica \\
\hline Habilidades de investigación \\
\hline Capaciadad de aprender \\
\hline Capaciadad de adaptación a nuevas situaciones \\
\hline Capaciadad de generar ideas nuevas (creatividad) \\
\hline Liderazgo \\
\hline Habilidad de trabajo autónomo \\
\hline Iniciativa y espíritu emprendedor \\
\hline Preocupación por la calidad \\
\hline Motivación de logro \\
\hline
\end{tabular}

Fuente: (Carazo et al., 2015) 
Así mismo, las experiencias de debate se han desarrollado con un formato de competición dialéctica entre grupos reducidos de alumnos (Carazo et al., 2015), con el objeto de combatir dialécticamente sobre un aspecto del contenido docente y, en ocasiones, con referencias a temas actuales de la sociedad (Olaso González et al., 2021), donde se expresan diferentes puntos de vista y que sirven como evaluación de la adquisición de contenidos por parte del profesorado.

Una de las limitaciones que tienen estos espacios de debate es que la participación se reduce al alumnado que presenta y discute los temas, por lo que, en aulas con un número elevado en el alumnado, se limita la competencia de participación.

En el curso 2020-2021, las condiciones académicas extraordinarias derivadas de la situación de prevención de la salud en la comunidad educativa universitaria ante la pandemia del coronavirus SARSCoV2 (Organization, 2020) han mostrado las brechas de acceso a la tecnología (García-Peñalvo, 2020).

Esta situación ha provocado cambiar la estrategia de enseñanza, pasando de una docencia presencial a otros modos híbridos con una parte o todo el alumnado siguiendo las actividades lectivas y prácticas "online". Además, las limitaciones sanitarias exigidas a raíz de la actual pandemia del COVID19 hacen complicado llevar a la práctica la metodología basada en debates presenciales. Por tanto, las nuevas condiciones sobrevenidas han obligado a diseñar nuevos espacios de formación del profesorado universitario (Suárez, 2020) y ha requerido el aprendizaje e implementación de estrategias didácticas y tecnológicas para así poder rediseñar los espacios y tiempos dentro de su labor docente.

El surgimiento de plataformas de videoconferencia ha posibilitado la adaptación de las universidades a estos formatos de docencia híbridos en los que una parte del alumnado siguen las clases por internet. Teniendo en cuenta estas necesidades, se ha planteado la oportunidad de aunar el desarrollo de espacios de debate utilizando como canal las herramientas de soporte on-line.

Existen pocas experiencias sobre el uso de los debates online en el entorno universitario, en el que se han utilizado de manera síncronas (por ejemplo Martin, 2006); o asíncronas con herramientas tipo foro de discusión (por ejemplo Mira et al., 2012). El debate virtual es considerado como: "un instrumento innovador y creativo en los procesos de comunicación, argumentación, negociación, interacción y aprendizaje colaborativo en los entornos educativos de educación superior (Hurtado et al., 2011), p. 175).

Este tipo de estrategias se pueden englobar en la metodología de aprendizaje colaborativo asistido por ordenador (CSCL o Computer Supported Collaborative Learning) que surge de prácticas de trabajo en grupo donde los integrantes cumplen unos roles complementarios para llegar a una meta en común (Han \& Hill, 2007). El resultado del aprendizaje es el desempeño de una tarea común a través de un entorno (en este caso virtual) que permite la interacción cooperativa.

Esta experiencia innovadora puede ayudar a implementar otra forma de estrategia docente en el ámbito universitario.

\section{Objetivo}

El objetivo del presente estudio es desarrollar una actividad de debate on-line de manera síncrona con grupos reducidos sobre un contenido de una asignatura del Grado de Criminología de la Universidad de Salamanca, a través de la aplicación zoom.us. De la misma manera, y como objetivos específicos se pretendía facilitar un espacio de reflexión para fomentar el proceso de enseñanza-aprendizaje, fomentar la participación de todo el alumnado e introducir las TIC's en la docencia. 


\section{Desarrollo de la innovación}

La actividad planteada se centró en el desarrollo de un espacio de debates síncrono online en el contexto de una práctica de la asignatura Psicología Criminal perteneciente al $2^{\circ}$ curso del Grado de Criminología de la Universidad de Salamanca. Se utilizó la temática de: "la influencia de los videojuegos en la delincuencia", que correspondía con el contenido docente y se presumía un contenido por el que el alumnado podía posicionarse a favor o en contra de manera sencilla.

Se utilizó la plataforma de videoconferencias zoom.us. Se eligió esta plataforma porque permite crear salas virtuales de una manera sencilla y está dentro de las utilidades de la Universidad de Salamanca. Las salas son espacios que crea el profesorado atendiendo al número de integrantes y método de distribución. Se utilizaron 4 salas de debate y se distribuyó al alumnado de manera aleatoria.

La semana antes de comenzar se habilitó en el espacio online de la asignatura un documento titulado "normas para el debate y criterios para la evaluación" con las instrucciones que marcaban el desarrollo de los debates, y que consistieron en:

- Antes de realizar la actividad el alumnado tenía que leer dos documentos vinculados a la temática que se quería debatir con argumentos a favor o en contra. el objetivo era que los textos proporcionaran una base teórica sobre el tema y sirvieran de incentivo para el desarrollo de posturas antagónicas.

- Una vez efectuada la convocatoria y con la presencia del alumnado se crearon 4 salas aleatorias. Se conectaron a la sesión 75 alumnos por lo que se conformaron 3 salas de 19 alumnos y 1 de 18 .

- Una vez creadas las salas, dentro de cada una, el alumnado elegirá 2 grupos de 3 personas que optarán por posicionarse a favor/en contra de la temática objeto de debate y deberán expresar su opinión acerca del mismo. Se tratará de expresar la opinión personal de cada uno e intentar convencer al otro de las ideas propias, con argumentos. El debate se basará en el respeto y no se utilizarán descalificaciones personales.

- En cada sala existirá un moderador (alumno) que será elegido al azar y que tendrá como misión el de conceder la palabra a los debatientes y asegurarse de que el debate de desarrolle en un ambiente de respeto.

- El debate durará 15 minutos. El alumnado seguirá en la sala correspondiente porque se tendrá que redactar un documento con las conclusiones del debate. En estas conclusiones podrán estar las opiniones más importantes que surjan en el debate y acuerdos a los que se hayan llegado. Este documento servirá como evaluación por parte del profesorado.

\subsection{Participantes}

Inicialmente se estuvieron convocados los 80 alumnos matriculados en la asignatura, pero únicamente asistieron $75(93,75 \%)$ La mayoría de los participantes eran mujeres (87,4\% frente a $22,6 \%$ hombres).

\subsection{Instrumentos}

Se elaboró un cuestionario $D G K R F$ para que el alumnado valorara la utilidad de la jornada de debate con la herramienta Google Forms. Dicho cuestionario tenía 10 ítems divididos en diferentes apartados (Tabla 2). Se formularon 2 preguntas sociodemográficas (sexo, edad), 4 preguntas sobre los contenidos y 6 preguntas sobre la metodología. Las preguntas que analizaron el contenido y la metodología estaban construidas en una escala Likert de 7 puntos siendo $1=$ de ninguna manera y $7=$ totalmente. 
Al final del cuestionario se solicitó que se valorara en una escala de 1 a 10 la experiencia y una pregunta cualitativa para que mostraran su opinión sobre las mejoras que se podrían realizar en la sesión de debate.

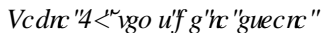

\begin{tabular}{|c|c|}
\hline Escala & Enunciado ítem \\
\hline Contenido & $\begin{array}{l}\text { La cantidad de documentos aportados ha sido suficiente para el desarrollo del debate } \\
\text { El tiempo empleado para el debate ha sido suficiente } \\
\text { Han aparecido opiniones a favor y en contra de la relación entre los videojuegos y la } \\
\text { delincuencia } \\
\text { El debate se ha efectuado con cordialidad }\end{array}$ \\
\hline Metodología & $\begin{array}{l}\text { Considero que el entorno utilizado es un entorno propicio para escuchar o poder } \\
\text { expresarse en experiencia de este tipo } \\
\text { La actividad ha sido entretenida } \\
\text { El documento sobre las conclusiones del debate es útil } \\
\text { La asignación de salas con un número reducido ha sido eficaz para la realización del } \\
\text { debate } \\
\text { La experiencia del debate online es una buena experiencia como complemento a las } \\
\text { explicaciones teóricas } \\
\text { Considero que el debate me ha ayudado a aprender el contenido de la asignatura }\end{array}$ \\
\hline
\end{tabular}

\section{Resultados}

Los resultados del cuestionario se muestran en la Tabla 3.

\section{DECDI ILC WDOWFRVGHFUSWWRV}

\begin{tabular}{|c|c|c|c|}
\hline Enunciado ítem & $\mathrm{N}$ & $\overline{\mathrm{X}}$ & SD \\
\hline ... ha sido suficiente para el desarrollo del debate & 75 & 6,3 & 1,09 \\
\hline ... ha sido suficiente & 75 & 6,21 & ,78 \\
\hline ... opiniones a favor y en contra de la relación. & 74 & 6,48 & ,94 \\
\hline ... ha efectuado con cordialidad & 75 & 6,47 & ,86 \\
\hline ... es un entorno propicio para escuchar o poder expresarse en experiencia de este tipo & 75 & 6,08 & 1,05 \\
\hline ... ha sido entretenida & 73 & 6,47 & 1,14 \\
\hline ... las conclusiones del debate es útil & 75 & 6,51 & 1,08 \\
\hline ... ha sido eficaz para la realización del debate & 75 & 6,30 & 1,15 \\
\hline ... es una buena experiencia como complemento a las explicaciones teóricas & 75 & 6,36 & 1,26 \\
\hline ... me ha ayudado a aprender el contenido de la asignatura & 75 & 6,28 & 1,45 \\
\hline
\end{tabular}

Atendiendo a la pregunta sobre la valoración de los debates, el alumnado ha valorado muy positivamente la innovación $(\overline{\mathrm{X}}=9 ; 01 ; \mathrm{SD}=, 98)$ 


\section{Conclusiones}

El presente estudio tenía como objetivo describir el desarrollo de una actividad de debate utilizando un formato online en una sesión síncrona con alumnado universitario. Ante la actual situación provocada por el COVID-19 y limitación de la presencialidad de las clases, el ejercicio de actividades no presenciales no debería ser un impedimento para suprimir las enseñanzas prácticas del alumnado universitario, ya que pueden servir para realizar actividades motivadoras, innovadoras y representar una oportunidad a la hora de fomentar sus habilidades comunicativas y argumentativas en torno al contenido docente. Esta propuesta elaborada para ser adaptada en escenario de semipresencialidad permite que se pueda poner en marcha sesiones prácticas en formato de debate con el alumnado.

La actividad realizada sobre los debates on-line ha despertado un gran interés entre el alumnado y ha supuesto una iniciativa interesante.

Se puede concluir que el alumnado ha estado satisfecho en la participación tanto con la metodología utilizada, como con el contenido elegido, siendo una actividad encomendada para otras asignaturas y cursos del Grado. Estos resultados son similares a las valoraciones efectuadas en otros estudios con el formato "clásico" de los debates y su desarrollo presencial (Mira et al., 2012; Olaso González et al., 2021).

Se ha podido comprobar que los debates on-line son una opción muy versátil y pueden servir como complemento de cualquier asignatura de manera transversal. El planteamiento efectuado ha mostrado que puede ser una herramienta eficaz para el desarrollo de seminarios y prácticas. Los temas y los contenidos a debatir pueden ser modificados de manera sencilla porque se pueden adaptarse a cualquier contenido.

Se puede afirmar que se han conseguido implementar los objetivos planteados en el proyecto ya que los debates han servido para incorporar el contenido docente a la docencia del espacio superior y se ha logrado incorporar las TIC's a la docencia en el entorno universitario, sobre todo, en los momentos actuales en los que se hace necesario y obligatorio diseñar estrategias de formación on-line.

Por último, hay que destacar el alto grado de satisfacción mostrado por el alumnado con la experiencia, que se traduce también en una satisfacción del profesorado implicado y que se añade a la motivación por desempeñar una docencia innovadora, pero sin perder el sentido de enseñanza-aprendizaje del contexto universitario. No obstante, el proyecto ha tenido algunas dificultades, sobre todo a la hora de manejar un grupo de alumnos tan grande en un contexto on-line. En futuras ediciones habrá que estudiar esta y otras dificultades que han aparecido, de cara a mejorar en la estrategia docente.

Así mismo, habrá que estudiar si este tipo de experiencias tienen algún efecto en el aprendizaje de los alumnos y en las puntuaciones obtenidas en la evaluación de la asignatura por lo que futuras investigaciones podrían avanzar en el diseño de este tipo de análisis.

\section{Referencias}

BENGOCHEA, L., \& MEDINA, J. A. (2013). El papel de los videotutoriales accesibles en el aprendizaje del futuro. Actas V Congreso Internacional sobre Aplicación de Tecnologías de la Información y Comunicaciones Avanzadas, 8087.

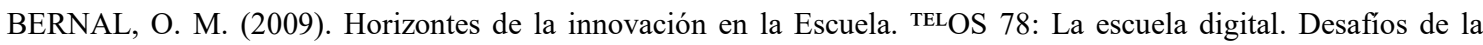
innovación educativa, 78,53 . 
CARAZO, R. M. A., IGLESIAS, I. B., \& SANZ, D. M. (2015). El “debate universitario” en la práctica de la asignatura Gestión de Empresas II del Grado de Relaciones Laborales y Recursos Humanos de la Universidad del País Vasco. Trabajo: Revista iberoamericana de relaciones laborales, 33, 45-71.

ESTEBAN GARCIA, L., \& ORTEGA GUTIERREZ, J. (2017). El debate como herramienta de aprendizaje. Jornadas de Innovación e Investigación Docente.

ESTEVE, F. (2016). Bolonia y las TIC: De la docencia 1.0 al aprendizaje 2.0. La cuestión universitaria, 5, 58-67.

GARCIA-PEÑALVO, F. J. (2020). El sistema universitario ante la COVID-19: Corto, medio y largo plazo.

HAN, S. Y., \& HILL, J. R. (2007). Collaborate to learn, learn to collaborate: Examining the roles of context, community, and cognition in asynchronous discussion. Journal of Educational Computing Research, 36(1), 89-123.

HURTADO, M. V., RAMOS, R., TRIGUEROS, E., BENGHAZI, K., NOGUERA, M., \& RODRIGUEZDOMINGUEZ, C. (2011). Entorno de interacción colaborativa mediante debate virtual. IEEE-RITA, 6(4), $175-182$.

MACEIRAS, R., CANCELA, Á., \& GOYANES, V. (2010). Aplicación de nuevas tecnologías en la docencia universitaria. Formación universitaria, 3(1), 21-26.

MARQUES GRAELLS, P. (2001). Algunas notas sobre el impacto de las TIC en la universidad. Educar, 28, $083-098$.

MARTIN, M. D. M. B. (2006). Desarrollo de una metodología docente para entornos virtuales. Revista Iberoamericana de Educación, 39(2), 1.

MIRA, J. B., PleguezUElOS, P. Z., RAMIREZ, L. V., RODRIGUEZ-CANO, C., VILA, R. R., JOVER, J. T., SERRANO, M. C., MARHUENDA, J. S., RAMOS, S. Á., \& ANDRES, S. M. (2012). El debate virtual como herramienta para conocer el pensamiento del alumno. X Jornadas de redes de investigación en docencia universitaria. La participación y el compromiso de la comunidad universitaria", ISBN, 978-984.

MORCHON GARCIA, R., SIMON MARTIN, F., \& GONZALEZ MIGUEL, J. (2016). Implantación de Kahoot como aplicación web en los seminarios de la asignatura de Parasitología en el Grado de Biología.

OLASO GONZALEZ, G., ROMA MATEO, C., \& PIQUERAS NEBOT, M. (2021). DEBATMITAL 2.0: 2a Edición de los debates sobre mitos en Alimentación. IN-RED 2020: VI Congreso de Innovación Educativa y Docencia en Red, 412-420.

ORGANIZATION, W. H. (2020). World Health Organization coronavirus disease (COVID-19) dashboard.

ORIHUELA GALLARDO, F., \& SIERRA-CASANOVA, C. (2020). Una experiencia de innovación docente: El debate académico en Administración de Empresas.

SUAREZ, N. (2020). Formación docente universitaria y crisis sanitaria COVID-19. CienciAmérica, 9(2), 109-114.

WIELENGA, D., \& MELISSE, R. (2000). Proving competence: Integrative assessment and web-based portfolio system in a dynamic curriculum. Society for Information Technology \& Teacher Education International Conference, 112117. 